\title{
Fatal anaphylactic reaction to intravenous infusion of Ondansetron: a report of two cases
}

\author{
Bouraoui OUNI ${ }^{1 *}$, Nessrine Bensayed ${ }^{2}$, Neila Fathallah ${ }^{3}$, Raoudha Slim ${ }^{3}$, Haifa Regaieg ${ }^{4}$, Bechir \\ Achour ${ }^{4}$, Yosra Benyoussef ${ }^{4}$, Abderrahim Khélif ${ }^{4}$, Chaker Ben Salem ${ }^{5}$ \\ ${ }^{{ }_{1} 1}$ Pharmacovigilance Regional Centre régionale de pharmacovigilance paris saint-antoine \\ ${ }^{2}$ Department of hematology, Farhat Hached hospital, Sousse 4000, Tunisia \\ ${ }^{3}$ Department of Pharmacovigilance, Medicine University of Sousse, Sousse University, Sousse 4000, \\ Tunisia \\ ${ }^{4}$ Department of hematology, Farhat Hached hospital, Sousse 4000, Tunisia \\ ${ }^{5}$ Department of Pharmacovigilance, Medicine University of Sousse, Sousse University, Sousse 4000, \\ Tunisia
}

Received: 02 November, 2017; Accepted: 05 December, 2017; Published: 20 December, 2017

*Corresponding author: Bouraoui OUNI, Pharmacovigilance Regional Center, Saint Antoine Hospital. Paris, France, Tel: +33-1.4347.5469; E-mail: elounibouraoui@yahoo.fr

\section{Abstract}

Ondansetron is a widely used antiemetic drug. Hypersensitivity to ondansetron is rare and fatal anaphylactic reactions to ondansetron have not been reported previously. Herein, we report two cases of anaphylactic reaction to ondansetron leading to death although adequate supportive therapies were quickly started.

Anaphylactic reactions to drugs manifests as respiratory distress due to laryngeal edema and intense bronchospasm that may be often followed by vascular collapse and shock. It is potentially fatal and prior exposure to an antigen is required for IgE dependent anaphylactic reactions. Our reported cases had a fatal outcome after they developed the anaphylactic reaction although they did receive ondansetron earlier in the previous courses of chemotherapy. In our cases, earlier doses have probably led to the sensitization of our patients.

Management of the anaphylactic signs and symptoms requires both supportive and preventive approaches. In view of life threatening anaphylactic/anaphylactoid reactions developing after ondansetron injection, high caution is required especially in patients with history of hypersensitivity reactions. Clinicians should be more cautious while using this antiemetic drug.
\end{abstract}

\section{Introduction}

Ondansetron is a selective 5 hydroxy-tryptamine 3 (5-HT3) receptor antagonist widely used as an effective antiemetic drug especially for the prevention of chemotherapy-induced nausea and vomiting [1]. The efficacy of ondansetron has been reported in several studies and it is reported to be a well-tolerated drug [2]. The most common side effects reported to ondansetron are headaches, diarrhea, constipation, fever and dizziness [3-5]. Anaphylactic reaction to ondansetron is rarely reported $[6,7]$.
To the best of our knowledge, fatal anaphylactic reactions to ondansetron have not been reported previously. Herein, we report two cases of anaphylactic reaction to ondansetron leading to death although adequate supportive therapies were quickly started.

\section{Results}

\section{Case 1}

A 32-year-old woman with no medical history was admitted to our department to undergo an induction course of chemotherapy for newly diagnosed acute myeloblastic leukemia. After the first injection of ondansetron, the patient immediately became hypotensive with a blood pressure of $80 / 40 \mathrm{mmHg}$. There was no accompanying urticaria, angioedema or respiratory distress, well managed with fluid infusion and hydrocortisone. The patient continued her chemotherapy and ondansetron without incidents. However, after the twentieth dose of ondansetron she claimed of dizziness, breath shortness and hypotension of $60 / 30 \mathrm{mmHg}$, dyspnea and tachycardia (150 bpm/min), without skin rash, edema or bronchospasm. Infusion of IV fluid and a bolus of dexamethasone were quickly administered. However, the hypotension persisted, 14 bolus of adrenaline $(0.1 \mathrm{mg})$ followed by continuous infusion of both adrenaline and noradrenaline in the purpose to enhance blood pressure were administered. Unfortunately, the patient died in a context of cardio respiratory arrest after 20 minutes of resuscitation. According to the Naranjo's causality assessment scale, the adverse event was 5 indicating "probable" reaction to ondansetron [8]. 


\section{Case 2}

A 34-year-old woman was scheduled to undergo chemotherapy for newly diagnosed hyperleuckotysis and tumor acute $\mathrm{T}$ lymphoblastic leukemia according to HyperCVAD chemotherapy which consists of two combinations of drugs (courses A and B) given in alternance. Since then, she received two courses (A) of cyclophosphamide, vincristine, dexamethason and doxorubicine and one B courses containing high dose methotrexate, cytarabine, arabinoside, in association with ondonsetron to prevent vomiting. All courses were received without incidents. During the fourth cycle (B), and during the first ondosetron infusion on this cycle, she immediately experienced flushing of her face and cutaneous itching followed by shortness of breath with chest pain, severe hypotension $(60 / 40 \mathrm{mmHg})$ and tachycardia. This hypotension was unresponsive to large intravenous fluid boluses and dexamethasone injections (8 $\mathrm{mg}$ ). Repeated adrenaline boluses and a noradrenaline infusion in association with adrenaline nebulization was also administred. Unfortunately she developed acute respiratory distress requiring intubation. Two hours later, the patient died in a context of refractory cardio respiratory arrest. According to the Naranjo's causality assessment scale, the adverse event was 6 indicating "probable" reaction to ondansetron [8].

\section{Discussion}

The main concern of patients receiving anticancer drugs is nausea and emesis because of release of serotonin from the enterochromaffin cells of the small intestine, which may stimulate the vagal afferent pathways through (5-HT3) receptors and so initiate the vomiting reflex. 5-HT3 selective antagonists such as ondansetron are widely used in cancer chemotherapy patients to prevent nausea and vomiting. Ondansetron is generally a well tolerated drug [3]. Life-threatening adverse reactions have been uncommonly reported, and may be secondary to coronary vasospasm, hypotension and generalized tonic-clonic seizures $[3,4,9]$. The incidence of anaphylaxis to ondansetron is low and fatal evolution is exceptional $[1,10-13]$. This has prompted some authors to suggest that ondansetron's use should be restricted in cases of anaphylaxia [14].

Anaphylactic reactions to drugs manifests as respiratory distress due to laryngeal edema and intense bronchospasm that may be often followed by vascular collapse and shock [15]. It is generally thought that prior exposure to an antigen is required for IgE dependent anaphylactic reactions [15]. Our reported cases had a fatal outcome after they developed the anaphylactic reaction although they did receive ondansetron earlier in the previous courses of chemotherapy. In our cases, earlier doses have probably led to the sensitization of our patients. In the report of Dermir et al, anaphylactic reaction to ondansetron occurred on the 56th dose albeit the patient received earlier doses without incidents [1]. Anaphylaxia also occurred after many administrations of ondansetron in the reports of Frigerio et al and Kossay et al $[11,14]$.
Many anaphylactic reactions may present without any usual features of allergy such as itching, flashing, edema or bronchospasm. The reaction may solely consist of marked hypotension, as was the case in our two patients [6]. In our first report, hypersensitivity reaction should be considered while the patient witnessed hypotension after the first injection. Clinicians should be aware of the risk of anaphylaxia secondary to ondansetron solely in the presence of hypotension or other manifestations of anaphylaxia even during the first infusions of the drug.

The exact mechanism of the adverse reaction to ondansetron is unclear. 24 reports to the Spontaneous Reporting System of the Food and Drug Administration (FDA) of anaphylactoidanaphylactic reactions in association with ondansetron injection were analysed [10].

These reactions may have been secondary to chemotherapy, but follow-up with persons who reported the domestic cases indicated that patients experienced no adverse events after being rechallenged with the same chemotherapeutic agents without ondansetron [10]. This finding suggests that intravenous ondansetron was the responsible cause. In our patients, ondansetron-induced anaphylactic reaction was probable according to the Naranjo probability scale. Ondansetron hypersensitivity is based on clinical results only, there are no confirmations of the diagnosis by highly sensitive and specific in vitro tests. We could perform skin tests in our patients, however the evolution was rapidly fatal.

Some authors suggest that anaphylaxis may be a class effect [16] while some others think it is drug specific effect [14] as ondansetron and tropisetron share indole heterocycle, while granisetron does not have indole ring [6].

Management of the anaphylactic signs and symptoms requires both supportive and preventive approaches [17]. Although our two patients received adrenaline, noradrenaline and steroids during the acute phase, anaphylactic reaction was severe and our patients unfortunately died.

\section{Conclusion}

Ondansetron is a wide used antiemetic drug. Rarely, anaphylactic reaction may occur. In view of life threatening anaphylactic/anaphylactoid reactions developing after ondansetron injection, high caution is required especially in patients with history of hypersensitivity reactions. Clinicians should be more cautious while using ondansetron, especially when the drug is used in an out-of hospital set-up where prompt treatment of the reaction may not be possible [7].

\section{References}

1. Demir HA, Batu ED, Yalçın B, Civelek E, Saçkesen C, Büyükpamukçu M. Anaphylactic reaction owing to ondansetron administration in a child with neuroblastoma and safe use of granisetron: a case report. J Pediatr Hematol Oncol. 2010;32(8):e341-342. Doi: 10.1097/ MPH.0b013e3181ea214a 
2. Jones AL, Hill AS, Soukop M, Hutcheon AW, Cassidy J, Kaye SB, et al Comparison of dexamethasone and ondansetron in the prophylaxis of emesis induced by moderately emetogenic chemotherapy. Lancet. 1991;338(8765):483-487.

3. Sharma A, Raina V. Generalised seizures following ondansetron. Ann Oncol. 2001;12(1):131-132.

4. Sargent AI, Deppe SA, Chan FA. Seizure associated with ondansetron. Clin Pharm. 1993;12(8):613-615.

5. Patel A, Mittal S, Manchanda S, Puliyel JM. Ondansetron-induced dystonia, hypoglycemia, and seizures in a child. Ann Pharmacother. 2011;45(1): e7. Doi: 10.1345/aph.1P332

6. Fernando SL, Broadfoot AJ. Ondansetron anaphylaxis: a case report and protocol for skin testing. Br J Anaesth. 2009;102(2):285-286. Doi: $10.1093 / \mathrm{bja} / \mathrm{aen} 376$

7. Mehra KK, Gogtay NJ, Ainchwar R, Bichile LS. Hypersensitivity to intravenous ondansetron: a case report. J Med Case Rep. 2008;2:274 Doi: 10.1186/1752-1947-2-274

8. Naranjo CA, Busto U, Sellers EM, Sandor P, Ruiz I, Roberts EA, et al. A method for estimating the probability of adverse drug reactions. Clin Pharmacol Ther. 1981;30(2):239-245.

9. J Lee. Ondansetron and chest pain. Emerg Med J. 2005;22(1):78.

10. Chen M, Tanner A, Gallo-Torres H. Anaphylactoid-anaphylactic reactions associated with ondansetron. Ann Intern Med. 1993; 119(8):862.

11. Kossey JL, Kwok KK. Anaphylactoid reactions associated with ondansetron. Ann Pharmacother. 1994;28(9):1029-1030. Doi: $10.1177 / 106002809402800906$
12. Ross, Allison Kinder, Ferrero-Conover, Deborah. Anaphylactoid reaction due to the administration of ondansetron in a pediatric neurosurgical patient. Anesth Analg 1998;87(4):779-780. Doi: 10.1213/00000539-199810000-00007

13. Goyal P, Paramesh K, Puranik S, Proctor M, Sanghvi M. Delayed diagnosis of anaphylaxis secondary to ondansetron: A case report. Eur J Anaesthesiol. 2016;33(2):146-147. Doi: 10.1097/ EJA.0000000000000372

14. Frigerio C, Buchwalder PA, Spertini F. Ondansetron: reasons to be restrictive. Lancet. 1996;347(9013):1484-1485.

15. Brockow K, Przybilla B, Aberer W, Bircher AJ, Brehler R, Dickel H, et al. Guideline for the diagnosis of drug hypersensitivity reactions: S2K-Guideline of the German Society for Allergology and Clinical Immunology (DGAKI) and the German Dermatological Society (DDG) in collaboration with the Association of German Allergologists (AeDA), the German Society for Pediatric Allergology and Environmental Medicine (GPA), the German Contact Dermatitis Research Group (DKG), the Swiss Society for Allergy and Immunology (SGAI), the Austrian Society for Allergology and Immunology (ÖGAI), the German Academy of Allergology and Environmental Medicine (DAAU), the German Center for Documentation of Severe Skin Reactions and the German Federal Institute for Drugs and Medical Products (BfArM). Allergo J Int. 2015;24(3):94-105. Doi: 10.1007/s40629-015-0052-6

16. Tolan MM, Fuhrman TM, Tsueda K, Lippmann SB. Perioperative extrapyramidal reactions associated with ondansetron. Anesthesiology. 1999;90(1):340-341.

17. Beyer K, Eckermann O, Hompes S, Grabenhenrich L, Worm M. Anaphylaxis in an emergency setting - elicitors, therapy and incidence of severe allergic reactions. Allergy. 2012;67(11):1451-1456. Doi: $10.1111 /$ all.12012 